\title{
Challenges in Developing Effective Clinical Decision Support Systems
}

\author{
Kamran Sartipi, Norman P. Archer and Mohammad H. Yarmand \\ McMaster University \\ Canada
}

\section{Introduction to CDSS}

Decision making is one of the most important and frequent aspects of our daily activities. Personal decisions display our characteristics, behavior, successes, failures, and the nature of our personalities. These decisions affect us in different ways, such as reasoning style, relationships, education, purchasing, careers, investments, health, and entertainment. The effectiveness of such decisions is affected by our age, knowledge, environment, economic status, and regulations. Our business related decisions are influenced by our knowledge, experience, and the availability of supporting systems in terms of employed processes, standards and techniques. Due to the importance of decision making, different technologies have been developed to help humans make effective decisions in the shortest time. Current advances in Information and Communication Technology (ICT) have revolutionized the way people communicate, share information, and make effective decisions.

Decision making is a complex intellectual task that uses assistance from different resources. In the past, such resources were restricted to personal knowledge, experience, logic, and human mentors. However, the norms of current society and existing technologies have enhanced critical decision making. Educational systems are not restricted to physical classrooms any more; on-line education is gradually taking over. Knowledge about a technical domain can be obtained easily using Internet search engines and free on-line scientific articles. Mentorship has expanded from colleagues and friends to a large community of domain experts through subject-specific social networking facilities. Moreover, due to ubiquitous wireless communication technologies, such facilities are also accessible from small and remote communities. As a result, technology and different web-based tools (browse and search, document sharing, data mining, maps, data bases, web services) can be utilized as computing support for people, to help them make more knowledgeable and effective decisions.

The healthcare domain has recently embraced new information and communication technologies to improve the quality of healthcare delivery and medical services. This long overdue opportunity is expected to reduce high costs and medical errors in patient diagnosis and treatment; enhance the way healthcare providers interact; increase personal health knowledge of the public; improve the availability and quality of health services; and promote collaborative and patient-centric healthcare services. To meet demands arising from these improved services, new tools, methods, and business models must emerge.

Clinical Decision Support Systems (CDSS) are defined as computer applications that assist practitioners and healthcare providers in decision making, through timely access to 
electronically stored medical knowledge, in order to improve their medical practices. For example, with the recent increased focus on the prevention of medical errors, Computer-based Physician Order Entry (CPOE) systems enhanced by CDSS have been proposed as a key element in improving patient safety (Berner, 2007).

An "effective" CDSS must also take into consideration the working environment of the practitioners and care providers. Hence such a CDSS should: not interfere with professional authority; recognize the context of the user and adapt itself accordingly; manage different types of information and interruptions that may affect the physician; save operating cost and time; be easy to use; adhere to medical guidelines provided by evidence-based research and practice; and support a patient-centric and collaborative decision making environment.

Clinical Decision Support Systems (CDSS) are specialized forms of general Decision Support Systems (DSS) that have been applied in many other domains. Research in decision support systems for organizational decision making began in the late 1950s, and research in the more technical aspects started in the 1960s (Keen \& Morton, 1978). Scott Morton (1971) was one of the first researchers to coin the term decision support systems (Eom \& Kim, 2006). Since then, major advances in computer technology have contributed to the application of DSS in many different disciplines and problem domains. In particular, advances in information technology infrastructure, data processing, microcomputers, networks, and human computer interactions have influenced DSS developments. Use of the Internet has enhanced DSS in terms of efficiency, widespread usage, and the employment of typical web browsers as user interface components. Recent advances in wireless communication technology and mobile devices have resulted in many new applications for decision support systems in daily activities (Shim et al., 2002).

The structure of this chapter is as follows. Section 2 provides an overview of different techniques and standards for representing clinical knowledge and information, with an emphasis on international standards such as HL7. Section 3 explores the nature of data mining techniques in assisting clinicians to diagnose illnesses and communicating the results of data mining. Section 4 discusses the influence of modern technologies and ad hoc web-application integration techniques to make collaborative decisions. Section 5 discusses the importance of user context and customizable software agents at the client platform. Section 6 provides a set of approaches to evaluate the success or failure of existing techniques, with a focus on business aspects and user adoption. In Section 7 the authors propose some research ideas that might contribute to the future development of more effective and acceptable clinical decision support systems. Section 8 provides several models and techniques from different fields that are used to support CDSS. Finally, Section 9 summarizes conclusions from this chapter.

\section{Clinical knowledge and information representation}

In a nutshell, a decision support system consists of the following components: i) knowledge base to store, maintain and retrieve knowledge from the relevant domain; ii) inference engine to retrieve the relevant knowledge from the knowledge base and interpret the knowledge to infer a decision; and iii) user support to interact with the user in a meaningful and natural way, with operations for data entry, representation, and result output. Such a system can also be improved by adding a history of the previous decisions, which is dynamically updated when new decisions are made.

We will discuss knowledge representation in this section. The inference engine is represented by different models and techniques that will be discussed in Section 8 . The user support component is mostly designed with web-based Graphical User Interfaces (GUI); however 
since this component has a major impact on the effectiveness of a CDSS, it requires particular attention from the research community.

Knowledge should be represented formally so that it can be efficiently processed. Such representation should be: human and machine readable; accurate in specifying domain knowledge; and portable and reusable among organizations (Verlaene et al., 2007). In general, knowledge representation methods can be categorized as declarative (using propositions and sentences), and procedural (explicitly defining the actions to be taken) (Aleksovska-Stojkovska \& Loskovska, 2010).

According to (Kong et al., 2008) four categories of knowledge representation are: i) Logical conditions: where variables and their valid ranges are provided and variable values are verified against their ranges; Boolean operators are used to specify more complicated cases. ii) Rules: expressed by if-then-else statements which emulate human reasoning processes; nesting statements are used for more complex cases. iii) Graphs: including decision trees and artificial neural networks. iv) Structures: high level categorization of relevant knowledge that allows focused observation of each of the healthcare sub-domains.

A major source of medical knowledge for decision making in a diagnosis or treatment process is the medical or clinical guidelines which have been used throughout the history of medicine. Modern clinical guidelines are developed based on rigorous studies of the medical literature and are based on consensus and evidence in medical research and practice. Such guidelines are represented as rules or flow charts and may include the computation algorithms to be followed. Guideline engines are used to execute the clinical guidelines in the context of Electronic Medical Record (EMR) systems. The GuideLine Interchange Format (GLIF) (Collaboratory, 2004) is a computer representation format for clinical guidelines that can be used for developing interoperable flow-based guidelines to be executed by such engines.

Another source of medical knowledge is the clinical terminology systems which allow healthcare professionals to use widely agreed sets of terms and concepts for communicating clinical information among healthcare professionals around the world for the purposes of diagnosis, prognosis, and treatment of diseases. A clinical terminology system facilitates identifying and accessing information pertaining to the healthcare process and hence improves the provision of healthcare services by care providers. The (Systematized Nomenclature of Medicine Clinical Terminology (SNOMED CT), 2011) is a comprehensive clinical terminology system that provides clinical content and expressiveness for clinical documentation and reporting. It can be used to code, retrieve, and analyze clinical data. The terminology is comprised of concepts, terms and relationships with the objective of precisely representing clinical information across the scope of healthcare. SNOMED CT uses healthcare software applications that focus on the collection of clinical data, linking to clinical knowledge bases and information retrieval, as well as data aggregation and exchange.

A number of tools exist that support knowledge construction during the CDSS development process. Four important tools are introduced here. UMLS - Unified Medical Language System is a repository of biomedical vocabularies which integrates over 2 million names for some 900000 concepts from more than 60 families of biomedical vocabularies, as well as 12 million relations among these concepts (Bodenreider, 2004). Protege is an ontology editor and knowledge-base framework that provides a suite of tools to construct domain models using frames and the Web Ontology Language (OWL) (Protege website, 2011). GLARE is used to acquire, represent, and execute clinical guidelines. It provides consistency checking and temporal and hypothetical reasoning (Anselma et al., 2011). PROforma is a formal knowledge representation language for specifying clinical guidelines in a machine executable format. 
Each guideline is expressed as a set of tasks, where a task can be of type: plan - contains any number of tasks; decision - taken at a point where options are presented; action: medical procedure; or inquiry - request for further information (Open Clinical: PROforma website, 2011).

\subsection{HL7 v3 reference information modeling}

Health Level Seven (HL7) (Health Level Seven official website, 2011) is an international community of healthcare experts and information scientists collaborating to create standards for the exchange, management and integration of electronic healthcare information. HL7 also refers to internationally accepted standards for healthcare information. Over the two decades since its inception, HL7 has undergone an evolutionary process starting from version 2.1 to its current version 3 (v3). HL7 v3 was a complete overhaul of its predecessor and was designed with consistency and comprehensive coverage in mind. It supports a wide range of areas such as patient care, patient administration, laboratory, pharmacy, diagnostic imaging, surgical procedures, insurance, accounting and clinical decision support systems. While all these topics are related, each of them has unique features and information requirements that need to be addressed by the standard. Furthermore, HL7 v3 uses several standard clinical terminology systems such as SNOMED and LOINC to represent information content. HL7 v3 uses Reference Information Model (RIM), a large class diagram representation of the clinical data. HL7 v3 applies object-oriented development methodology to RIM and its extensions to create standard message content.

The HL7 refinement process uses RIM class diagrams, HL7-specified vocabulary domains, and data type specifications, and applies refinement rules to these base standards to generate information structures for HL7 v3 messages. The message development process consists of applying constraints to a pair of base specifications, i.e., HL7 RIM and HL7 Vocabulary Domains, and the extension of those specifications to create representations constrained to address a specific healthcare requirement.

We now refer to the artifacts generated in the refinement process. The Domain Message Information Model (D-MIM) is a subset of RIM that includes a fully expanded set of class clones, attributes and relationships that are used to create messages for any particular domain. The Refined Message Information Model (R-MIM) is used to express the information content for one or more messages within a domain. Each R-MIM is a subset of the D-MIM and contains only those classes, attributes and associations required to compose the set of messages. Hierarchical Message Description (HMD) is a tabular representation of the sequence of elements (i.e., classes, attributes and associations) represented in an R-MIM. Each HMD produces a single base message template from which the specific HL7 v3 message types are drawn (Health Level Seven Ballot, 2011; HL7, 1999).

\section{Data mining and interoperability in CDSS}

In this section, we describe a set of related techniques that demonstrate the role of data mining in discovering important hidden patterns among clinical data. In this case, association mining using concept lattice analysis discovers groups of diseases, symptoms, and signs that are highly associated. These groups assist the physician in disease diagnosis process. We further discuss how such important patterns of relationships (we call them mined-knowledge) can be transported to the point of use, where such knowledge can be incorporated into decision support systems to enhance physician decision making activity. We also present the supporting standards and infrastructure that allow such collaboration among heterogeneous systems. Data mining is the process of analyzing data from different perspectives to extract 
information and hidden patterns that are useful for planning and configuration purposes. Technologies from various domains such as statistics, data warehousing, and artificial intelligence support data mining activities. Chapter 3 in the book by (Berner, 2007) discusses the applications of data mining in CDSS.

\subsection{Concept lattice analysis in diagnostic process}

The following discussion is based on (Yousefi et al., 2009). The exploration nature of the association based data mining techniques (e.g., concept lattice analysis) in a diagnostic process simulates the normal process that a practitioner follows in clinical practice. On a daily basis, physicians encounter complex clinical scenarios where they compare a set of clinical observations in the form of symptoms and signs, with those they know based on their medical knowledge and experience, in order to make accurate disease diagnoses. In this context, the patient history from the EMR is also used as complementary information to clinical observations. The approach takes advantage of the automatic extraction of patterns from a patient EMR system using concept lattice analysis and uses a ranking mechanism to indicate the degree of relevancy of the clinical observation to each member of the identified group of diseases. Syndrome is a set of signs and symptoms which tend to occur together and reflect the presence of a particular disease. There are a large number of major clinical syndromes that can be modeled according to this technique. As a case study, the authors modeled a syndromic approach to Fever of Unknown Origin (FUO) due to its importance and complexity in the medical domain. There are a large number of cases with FUO which are undiagnosed despite hospitalization, costly paraclinic requests and invasive procedures. The authors have modeled FUO as an example of a major clinical syndrome. The case study modeled 45 diseases and 64 common symptoms and signs associated with FUO from a heavily cited medical reference by (Mandell et al., 2004). In this approach, a Concept Explorer tool (Formal concept analysis toolkit version 1.3, 2009) was used to illustrate the context table and concept lattice which present the relations among the diseases and their associated symptoms and signs in a large lattice consisting of 499 concept nodes. The concept lattice can be used as follows. During a patient visit, the physician observes and records the patient symptoms and signs, and consults the patient EMR to obtain other possible symptoms that may be relevant to the current visit. The tool then compares this set of symptoms and signs with those of different concepts in the concept lattice. The concepts with the highest overlap of symptoms and signs are then retrieved and a ranked list of the diseases within the concepts is presented to the physician. The physician uses his/her discretion to identify which disease within the provided concepts would be the best match with the patient's situation. The authors discuss a case of an elderly female patient who had a fever for four days with other symptoms such as anorexia, malaise, non productive cough, night sweats, and chill. Using the approach described, four diseases were suggested to the physician: Tuberculosis, Sarcoidosis, Lymphoma, and Recurrent Pulmonary Emboli, where the latter was found to have the highest overlap with the patient's symptoms.

\subsection{Interoperability of mined-knowledge for CDSS}

The details of this technique were presented in (Sherafat \& K. Sartipi, 2010). Currently, decision making knowledge within most guideline modeling languages are represented by basic logical expressions. However, the results of data mining analyses from healthcare data can be employed as a source of knowledge to improve decision making. A CDSS can interact with practitioners and electronic medical records systems to receive patient data as 
input and provide reminders, alerts, or recommendations for patient diagnosis, treatment, long-term care planning, and the like. A CDSS requires access to healthcare data and knowledge that are stored in data and knowledge bases. Since these repositories normally have diverse internal representations, data and knowledge interoperability are major issues. To achieve data interoperability, two systems that participate in data communication must use the same vocabulary set, data model, and data interpretation mechanism. On the other hand, knowledge interoperability refers to the ability of healthcare information systems to incorporate and interpret the knowledge that is produced in other systems. Here, we focus on encoding, sharing, and using the results of data mining analyses for clinical decision making at the point of care.

The proposed approach relies on adoption of standards to encode healthcare data and knowledge. In an off-line operation, existing healthcare databases (i.e., EMRs) are mined using different mining techniques to extract and store clinical mined-knowledge. In order to make this knowledge portable it is encoded in the form of a data mining model using a specialized XML-based standard, namely Predictive Model Markup Language (PMML) (DMG, 2010). Also, it is necessary for the patient data that are stored in EMR systems to be encoded using a specialized XML-based standard, namely Clinical Data Architecture (CDA) $(H L 7,2005)$ so the data can be ported between heterogeneous systems. At the point of care, a decision module accesses and operates on both data and knowledge in order to make patient-specific interpretations of the knowledge available to the healthcare practitioner. Within the CDSS we adopt a flow-oriented clinical guideline modeling language GLIF3 (Collaboratory, 2004) to specify the overall decision making process. In this context, at different states of the flow-oriented guideline the CDSS accesses patient data by querying the EMR. Moreover, to perform knowledge-based decision making, the CDSS supplies patient data to the decision modules and receives the results of applying mined-knowledge to the patient data. Finally, healthcare personnel receive comments, recommendations, or alerts through interaction with the CDSS system, allowing them to make more knowledgeable decisions based on system-provided information.

\subsection{Interoperability of clinical information and concept}

The details of this technique were presented in (Jayaratna \& Sartipi, 2009). A key objective of effective healthcare delivery is to facilitate seamless integration among heterogeneous applications, to provide a unified view of information to health practitioners and other stakeholders. Achieving such flawless integration requires interoperability among data sources serving the applications. This can only be achieved through standardization of information exchange and representation. The HL7 Reference Information Model (RIM) was introduced in Section 2.1. HL7 v3 based integration of systems requires an expert in medical domain who is also familiar with HL7 v3 standards and documentation. Hence, such an integration process is expensive, slow, and expert-based. In the following, we present a framework to support HL7 v3 message extraction for standard compliant integration projects, based on Semantic Web (SW) technologies.

We have observed that the existing HL7 domain model does not facilitate efficient discovery of HL7 v3 messages due to overlaps and disconnects among the domains. Therefore, we developed a more intuitive and finer categorization for HL7 domains, namely contexts, which consist of 50 contexts to represent areas of healthcare that superimpose well with actual healthcare transactions. Each HL7 v3 message was associated with a single context. Context acts as a key piece of metadata in the search tool. Next, we classified HL7 messages into a 
hierarchy of classes based on the purpose of the messages they convey. This classification has been designed to be intuitive and general enough so that it could also be used to formally express a clinical transaction. In the following, the steps for the message extraction process are discussed.

- Step 1: Integration Requirements Analysis. This step consists of: i) Storyboards which are a set of scenarios in the health domain written by health professionals in their own terminology. ii) Context extraction, where the tool searches storyboard text to create possible semantic maps between the Contexts and the words and phrases in the text. Within the tool, each Context has been annotated with Cognitive Synonyms that describe it. WordNet and SNOMED vocabularies were used to incorporate as many cognitive synonyms and phrases needed to describe each Context. iii) Identify transaction initiators, where each initiator starts a message in a sequence of messages that complete a transaction. Transaction initiators can be easily identified manually from storyboard text, while adhering to the obtained Contexts.

- Step 2: Structured transaction generation. Each transaction initiator is then structured according to the proposed transaction schema so it is in machine readable format.

- Step 3: Mapping. This step consists of: i) Message mapping, where structured transactions are entered into the tool, and the tool's advanced semantic search feature searches the main artifact repository to find a matching message. ii) Vocabulary mapping, which converts local terms into standard terminology codes for transmission. The tool integrates with terminology systems SNOMED and LOINC to search for the most appropriate code for a particular legacy clinical term. Data fields extracted during Step 1 are used as search criteria.

\section{Collaborative decision making}

In this section, we discuss the influence of modern technologies in social networks and ad hoc web application integration techniques to form focused groups of specialists to make collaborative decisions about a critically ill patient. Such an environment integrates different facilities for collecting patient records from the Electronic Health Record (EHR) system according to the case at hand and allows clinicians, nurses, and other support staff to communicate asynchronously through email, text messaging, and video conferencing. The platform will support conversations, collaborative decisions, etc. in a secure data center and will issue reminders and follow-ups to the group and the patient.

In current healthcare systems, patients are often not active participants in their treatment; instead they rely on the practitioner who guides the diagnosis and treatment process. This process is not particularly effective as the patient may not be interested in the process, but just in a favorable outcome, i.e., a successful treatment. With the advent and popularity of social networking, people tend to form groups with special interests and share information and knowledge. This allows different patients with the same health related subject of interest to form small communities to share their experiences, augment their knowledge, and mutually encourage themselves to be more involved in their treatment process (Bos et al., 2008). Current Personal Health Record (PHR) systems help patients to be more aware of their treatment processes and to obtain information about their health. However, future PHR systems will allow more patient involvement through improved user interfaces to access their own healthcare data and sophisticated services through new features empowered by Web 2.0 technologies (Oreilly Web 2.0 Books, 2011). 
A Mashup (Abiteboul et al., 2008) is a Web 2.0 technology which is gaining popularity for developing complex applications by combining data, presentations, and applications from different sources to create a new web application. The approach is new and is still being enhanced. However, the need for fast and easy development of ad hoc web based applications has caused a large number of Mashups to be developed that are organized into different categories (ProgrammableWeb Web site, 2011). Such characteristics make Mashups an ideal tool for non-critical web-based data management applications, since they can be developed in a short time, with very little programming skill. The goal of a Mashup is to provide a means to utilize a large number of web based applications and heterogeneous data sources in a unified representation. However, Mashups do not meet the hard constraints imposed by application domains that incorporate sensitive data, real-time operations, or mission critical tasks.

An example of a Mashup, namely MedickIT, is presented by (Abiteboul et al., 2008), and it consists of six components (or Mashlets). These Mashlets can be GUI-based components (i.e., widgets) or web services. The MedickIT consists of six Mashlets: an Electronic Health Record viewer, a map widget, a calendar, a medical search engine, an SMS, and a medical data analyzer. Such a Mashup allows a patient to access his/her medical data through the EMR; retrieve a doctor appointment from the calendar and drag it into the map to see the location of the doctor's office, or drag it into the SMS widget so that a phone call is initiated to remind the patient about forthcoming doctor appointments. Such a Mashup will allow the patient to gain more control over his/her health information. Depending on the needs of the patient, other combinations of Mashlets are feasible.

The (IBM Mash up Center, 2011) is an end-to-end enterprise Mashup platform that supports rapid assembly of dynamic web applications with management, security, and governance capabilities. It allows both nontechnical users and IT personnel to develop complex applications. In the case of collaborative healthcare delivery, an ad hoc case-specific team of local and remote professionals can be formed which consists of doctors, nurses, administrative and support staff. The team can communicate and brainstorm through the e-conferencing widget, access the patient's EMR record, obtain reports of the patient's risk factors by consulting with a CDSS tool, and make collaborative diagnostic decisions for the patient.

\section{Challenges in future CDSS}

In this section, we discuss some challenges that designers of future clinical decision support systems face. Such challenges include better interactions with users to understand their work context, and utilizing customizable computer agents in the client platform. In general, user-centric and collaborative features of CDSS systems impose higher levels of abstraction and more intelligent service-based computing at both the application and middle-ware layers.

\subsection{Customizable and context-based CDSS}

The current state of CDSS web applications is represented by the services that require extra knowledge and expertise from a normal user to take advantage of the available features and operations of these services. Given the large variety of web applications as Mashups and the tight time schedules of users, they will have to limit themselves to a minimum set of available service features. This is also the case in using other types of computerized systems such as automobile gadgets, home appliances and entertainment centers. In other words, the proper and efficient use of computerized systems (embedded or software based) requires an extended level of knowledge in different application domains. The user interaction capabilities of these systems tend to be sophisticated and hence these systems act as effective user assistants by 
providing different types of information to assist users in performing their tasks. However, domain knowledge and expertise are still needed by users.

The next generation of CDSS systems will become even more sophisticated when the required expertise is incorporated as part of the system's functionality. For example, instead of expecting users of a collaborative CDSS that uses a Mashup service to understand the details and the operational steps needed to use the specialized web application, the web service itself should act as an expert. This expert would consult with the user to provide an effective and customized use of operations according to the user's specific context information. This would provide an opportunity for the user to employ an expert software agent for managing web assets and performing the desired tasks with minimum effort and time. Such a software agent would support smart interactions with the system by the user. Such customizable software agents are resident at the client platform (as opposed to mobile agents that can move among different platforms) with a customizable architecture that receives a set of well-defined tasks in order to become expert and serve the user. The proposed customizable software agents would add to current traditional services, which receive a client's request for a service, perform the service at the provider's platform, and return the results to the user. In the following, the steps for using such a client-based customizable expert agent are presented:

- Step 1: identifying user context. Context refers to any information that can be used to characterize the situation of a service requester or provider. We define a context as a tuple: $<$ User, Role, User Location, Server Location, Time of Day, Team, Delegation, Requested Profile Status, Service Invocation Type, Requested Data Type, Login/Logout Event $>$. This context information is monitored dynamically to feed a database of context logs which will be used during the service selection.

- Step 2: selecting the required task. The user (e.g., a physician) asks for a specific task and the required expertise needed for assistance. By mining the context logs (Step 1) and consulting with a web registry, a client proxy obtains a list of relevant services to perform the task, and generates a list that ranks them according to their capabilities and any associated charges. The user then selects an appropriate service, which best matches with the situation. In this context, the web registry must possess a list of application domains such as: banking, insurance, healthcare, telephone, airline, government, etc.; as well as a list of tasks within each domain, such as: PHR viewer, medication administrator, medical data analyzer, and medical search engine, within the healthcare domain.

- Step 3: delegating expertise to the client. After selecting the required task, the client proxy retrieves the service descriptions of the selected service and invokes the service from the provider's platform. Instead of performing the requested task for the client, the provider will send a set of instructions to the client where the customizable expert agent will customize itself to serve the physician in an interactive clinical decision activity.

We have already applied the above architecture in several projects, including a customizable virtual remote nurse (Najafi et al., 2011), web service composition (Najafi \& Sartipi, 2010), and web service selection tasks.

\section{Evaluation of techniques, adoption, and success of CDSS}

The four key functions of CDSS were outlined by (Perreault \& Metzger, 1999), as follows: i) Administrative: supporting clinical coding and documentation, authorization of procedures, and referrals; ii) Managing clinical complexity and details: keeping patients on chemotherapy 
protocols, tracking orders, referrals follow-up, and preventive care; iii) Cost control: monitoring medication orders, avoiding duplicate or unnecessary tests; and iv) Decision support: supporting clinical diagnosis and treatment plan processes and promoting use of best practices, condition-specific guidelines, and population-based management. These functions are not necessarily logically separable, so they are addressed in a relatively all-inclusive manner in this section.

In some cases, a CDSS may not need to be justified through improved patient outcomes because these systems are designed to influence healthcare providers and it is necessary only to demonstrate changes in clinician performance (Balas \& Boren, 2007). But in many cases relationships between process and outcome is unclear (such as personal electronic medical records used by patients for self-management of certain chronic illnesses). However, in order to compete for scarce resources in the healthcare environment, developers must demonstrate the relevance of their systems to healthcare quality improvement and cost control. This requires evaluation approaches that are convincing to potential users, and focused on differences in the process or care outcomes involving the use of the CDSS (Balas \& Boren, 2007).

CDSS vendors often claim that their systems can directly improve clinical decisions. A wide variety of approaches and methodologies are available to assess these claims, ranging from controlled clinical trials to use of questionnaires and interviews with users. Techniques that are used should be based on fundamental principles and methods from cognitive science and usability engineering, where human computer interaction and usability in both laboratory and natural environments are examined. Methods can and should include the formative evaluation of systems during iterative development, and can also complement traditional summative assessment methods for completed systems (Kushniruk \& Patel, 2004). CDSS designers may prefer to use benchmark tests, surveys, and historical control comparisons (before-after studies) to indicate improvements in quality due to the use of a new system. But benchmark tests only measure a system's technical performance, and do not indicate the system's impact on processes or outcomes of care. User opinion surveys can only provide indirect information about system impact. Before-after studies may provide useful information, but analysis of databases or historical control groups of patients cannot replace planned clinical experimentation. Randomized Controlled clinical Trials (RCTs) are generally recognized as the gold standard for determining the efficacy of computerized information systems in patient care. There are many types of randomized clinical trials but the basic principles are the same: prospective and contemporaneous monitoring of the effect of a randomly allocated intervention (Balas \& Boren, 2007).

One dissenter is (Kaplan, 2001) who suggests that Randomized Controlled clinical Trials (RCTs) are not suited to determining whether and/or how systems will be used. In particular, since CDSS are not yet widely used, it is important to develop evaluation techniques that will determine why this is the case, even for systems that seem to offer a great deal of promise for clinical support. Kaplan proposes a $4 \mathrm{C}$ approach that focuses on communication, control, care, and context, an approach that can be used for evaluating other types of clinical systems. For a fuller understanding of system operations, it is important to investigate social, cultural, organizational, cognitive, and other contextual concerns that can increase the understanding of other influences that affect systems application development and deployment.

In a systematic review of controlled clinical trials of CDSS systems on physician performance and patient outcomes in 1998, (Johnston et al., 1994) studied 68 controlled trials, and found that 43 of the 65 that evaluated physician performance showed a benefit, and six of 14 
studies assessing patient outcomes found an improvement. Their basic conclusions were that CDSS can enhance physician clinical performance for drug dosing, preventive care, and other aspects of medical care, but not convincingly for diagnosis and that there was not yet sufficient evidence to determine the effects of CDSS on patient outcomes. In a 2005 followup review of 100 studies, (Garg et al., 2005) found that improved practitioner performance was associated with CDSS that automatically prompted users to activate the system, or when the study authors actually developed the CDSS. However, there were still not enough studies of patient outcomes available, so the impact on patient outcomes was not clear. RCTs do have limitations, since they can test only hypotheses about certain aspects of computer systems. RCT studies need to identify the conditions to be treated, interventions to be tested, and outcome variables to be measured. The results can then be regarded as specific, interpretable, and useful for practical purposes (Balas \& Boren, 2007).

Measuring and managing user attitudes toward various aspects of information systems is important in showing that computer systems are successful, since success is not possible without gaining the support of practitioners. Questionnaires can be used to measure user attitudes to the system. A critical success criterion for the usefulness of a system is how users react to various system aspects. Overall high satisfaction levels usually result in users adapting their activities to take advantage of the system. If satisfaction levels are low, users may actually become antagonistic and sabotage the system, or develop workarounds that avoid using the system.

Randomized controlled trials have shown that there are four generic information interventions that can make a significant difference in patient care (patient education, treatment planning, physician and patient reminders) (Balas et al., 1996). It is therefore important to incorporate these information services into any CDSS that will be used for primary care, in order to improve its effectiveness.

Following are three examples that demonstrate the diversity of methods for implementing and evaluating CDSS that all include the modern approach of using some form of a parallel development and evaluation process.

- (Trafton et al., 2010) developed and implemented a CDSS using iterative evaluation throughout system analysis, design, development, implementation, including simulation and in-clinic assessments of usability for providers followed by targeted system revisions. Volunteers that evaluated the system at particular times provided detailed feedback that was used to guide improvements in the graphical user interface, system content, and design changes that increased clinical usefulness, understandability, clinical workflow fit, and ease of completing recommended practices according to specific guidelines. These revisions led to improved CDSS usability ratings over time, including attention to other practice concerns outside the scope of the CDSS.

- One of the anticipated benefits from Computerized Physician Order Entry (CPOE) systems is the reduction of medication errors, but only a minority of hospitals have successfully implemented such systems. Physician resistance and frustration with such systems have been barriers to their use. An innovative approach to improve adoption and to realize the full benefits of such systems is to involve nurses in the order entry process in order to reduce physician data entry workload and resistance. (Kazemi et al., 2010) investigated whether a collaborative order entry method consisting of Nurse Order Entry (NOE) followed by physician verification and countersignature was as effective as a strictly Physician Order Entry (POE) method in reducing dose and frequency medication errors in a neonatal ward. They found a significant reduction in medication errors during the NOE 
period compared to the POE approach. The additional benefit to using such an approach is that physicians no longer have to participate in data entry, helping to overcome this barrier to CPOE use.

- A good example of modern CDSS development is provided in a paper by (Leslie et al., 2005). This work focused on the development and evaluation of a CDSS to assist physicians in treating patients with chronic heart failure, and provides definitive support for the concept of iterative evaluation during CDSS development (Trafton et al., 2010) combined with a multidisciplinary approach to organizational and social aspects of the system environment (Kaplan, 2001). The CDSS was developed after discussions with a multidisciplinary panel, and evaluation took place during three stages over a 6 month period that involved an editorial check, interviews with potential users, and educational meetings with users. The process resulted in several changes to the CDSS at different stages of evaluation and development. Trends were found when comparing the CDSS with paper guidelines. GPs scored less well but junior doctors and medical students appeared to improve their scores. $70 \%$ of the users indicated that the CDSS was more useful than written guidelines. Implementation barriers included lower computer literacy among GPs, a lack of complexity within the CDSS that could address non-medical needs of patients, and medical staff reluctance to consult guidelines during patient consultations.

\section{Research challenges in CDSS}

In this section, the authors propose some research ideas that they believe would contribute to the development of more effective and acceptable clinical decision support systems in future. Due to several limitations imposed by current economic and environmental situations world-wide, both the public and industry have raised their expectations in terms of the quality, performance and cost of future systems and equipment in different fields. Healthcare is no exception. In fact, given the recent increased adoption of IT by healthcare professionals we envision that promising changes in terms of efficiency and effectiveness of healthcare service delivery will happen in the near future. As suggested in this chapter, clinical decision support systems have already taken advantage of existing techniques and models from the computer science and information systems fields. However, the following research avenues need more exploration:

- Human Computer Interaction (HCI): current computer interfaces seem to be restrictive and less flexible than expected for seamless and natural interactions that clinicians might expect to use in daily practice when using clinical decision support. Therefore, HCI research plays a key role in making CDSS systems more effective and acceptable. HCI involves inter-disciplinary research covering computer vision, machine learning, network security, database design, artificial intelligence, multimedia technology, embedded computation, ergonomics and cognitive psychology. It includes the design, implementation and evaluation of interactive computing systems for human use (ACM SIGCHI Curricula for Human-Computer Interaction, 2011). The goal of HCI is to achieve natural interaction between humans and computers. Actions of users can be captured as inputs, including vision (e.g., body movement and hand gestures), audio (e.g., speech), smell, touch, and taste. Advances in context awareness and context mining technologies have made it possible to extract and analyze implicit inputs. These implicit outputs can be integrated with the user environment instead of interrupting the user, allowing users to concentrate on their work (Schmidt, 2002). 
- Security and privacy: healthcare systems that operate with sensitive clinical data require particular attention and procedures to ensure authorized access in order to prevent privacy and security breaches of clinical data residing in the CDSS. There has been a dramatic increase in reports of security breaches. In particular, since most healthcare systems (including CDSS) are web and service-based (e.g., Mashups), preserving the integrity, security and privacy of patient data has utmost importance. Therefore, a research challenge is to target security and privacy issues. As an example of such research, (Sandell, 2007) secures personal health data by proposing a framework which breaks CDSS into data gathering, data management, and data delivery functions. It then provides vulnerability factors and the required measures to protect data.

- Mobile CDSS: uses mobile devices or smart phones as the mediator between the healthcare provider (clinician, pharmacist, emergency staff) and the patient, so that the provider sends specific instructions and guidelines to the mediator. The mobile device then interacts with the patient to control his/her health condition according to the received instructions and guidelines. New advances in mobile communications allow remote areas and homecare to use a variety of remote health services. This opportunity has aroused much attention in the research and development of mobile healthcare (mHealth) and related services, such as Mobile CDSS (Tsumoto et al., 2005).

\section{Related techniques in CDSS}

Several models and approaches have been proposed for decision support systems. In particular, management science and operations research models have been used frequently. In this section, we briefly present the techniques that have been applied in health and medical domains (Berner \& Lande, 2007; Eom \& Kim, 2006; Shim et al., 2002). These techniques can be categorized as follows.

\section{Deterministic models}

- Linear programming: a method for finding the best solution for a given mathematical model satisfying certain constraints by maximizing or minimizing an objective function, subject to linear equality and inequality constraints. (Hershey, 1991) uses linear programming to find optimal clinical strategies when event probabilities are not known but their value ranges are available. Linear programming has also been used for an operating room planning problem which employs 0-1 linear programming (Testi \& Tànfani, 2009).

- Inventory models: designed to minimize inventory costs by setting optimal values for time to place an order and order quantity. Placing an order can be performed either at fixed time spots or decisions can be based on the condition or level of the inventory (Oh \& Hwang, 2005).

- Integer programming: a mathematical optimization problem and a special form of linear programming problems where variables are restricted to be integer. In (Eben-Chaime \& Pliskin, 1992) a mixed integer programming model is used to decide on the size and location of beds required in a given region, incorporating travel times into dialysis planning.

- Nonlinear programming: used to maximize or minimize an objective function subject to a system of equality and inequality constraints where either the objective function or some of the constraints are nonlinear. (Aspden et al., 1981) use a non-linear programming model 
to study the problem of distributing available healthcare resources (such as hospital beds or nurses) to assist health services planing.

- Dynamic programming: a problem solving method similar to divide-and-conquer with overlapping sub-problems that have optimal sub-structures. (Hall, 2010) uses dynamic programming models to investigate individual behaviors and their economic implications. This approach has been applied to healthcare spending, long-term care insurance, employment, entrepreneurial risk taking, and consumer debt.

\section{Stochastic models}

- Queuing: a technique for representing different types of queues to study their behavior. Certain performance measures are extracted as the result of queueing analysis. (Patrick \& Puterman, 2006) use a queueing model to increase the utilization of Computed Tomography (CT) scanning devices and reduce waiting times of patients with several priority levels.

- Markov: a stochastic model that is suitable for problems where the Markov assumption holds, i.e., problems that have memoryless properties. Problems may become intractable without this assumption. (Sonnenberg \& Beck, 1993) discuss applications of Markov models in CDSS. They introduce the generic class of problems suitable for modeling with Markov models and different evaluation methods such as matrix algebra, cohort simulation, or Monte Carlo simulation.

\section{Artificial Intelligence}

- Artificial neural networks: an information processing paradigm that is inspired by the structure and functional aspects of biological neural networks and the way they process information. A neural network consists of an interconnected group of processing elements (artificial neurons). Adaptive learning, self-organizations, and fault tolerance are among dominant features of this paradigm. (Mangalampalli et al., 2006) used the concept of neural networks to develop a decision-support system, that suggests medications for a gynecological disease, based on the primary and secondary symptoms of the disease.

- Genetic algorithms: an adaptive heuristic search algorithm that relies on the concept and process of natural evolution, selection, and genetics. This method is used in search spaces where little knowledge exists about the domain, the existing knowledge is difficult to encode, or mathematical models are not available. (Zellner et al., 2004) employed genetic algorithms to improve the performance of a logistic regression model in predicting the presence of brain neoplasia with magnetic resonance spectroscopy data.

- Game theory: mathematical modeling for games in which a player attempts to achieve a certain goal which is dependent on the choices of other players. Game theory offers strategies for increasing the probability of success. Solutions can be extended to cases where several players seek goals with multiple criteria (Parsons et al., 2002).

- Decision trees: visual and analytical tools that consist of three types of nodes: decision nodes, chance nodes, and end nodes. Decision trees are usually used for guidelines and are extensively used in CDSS. Examples can be found in (Critchfield \& Willard, 1986; Hazen et al., 1998; Sonnenberg \& Beck, 1993). 


\section{Practical}

- Simulation: a generic term that encompasses approaches using simulation for decision making. (Critchfield \& Willard, 1986) use Monte Carlo simulation techniques to model the uncertainty in the specification of decision tree probabilities. They apply their method to the clinical problem of anti-coagulation versus observation in combatting deep vein thrombosis during the first trimester of pregnancy. Their method provides decision analysts with tools to quantitatively evaluate the problem. In another work, simulation was used to determine the utilization of a cancer agency ambulatory care unit. This work analyzed the impact of operations, scheduling, and resource allocation on patient wait time, clinic overtime, and resource utilization (Santibanez et al., 2009).

- Visual interactive modeling: provides animated graphics for target applications. This model offers interaction facilities so users can explore the dynamics of an application in order to gain an understanding of its features (Au \& Paul, 1996).

\section{Conclusion}

The healthcare system is witnessing major changes, in terms of reengineering healthcare processes and policies which will affect all stakeholders, ranging from healthcare providers to policy makers to consumers. These changes will bring technologists and medical experts closer together and will provide a more homogeneous environment for effective collaboration of these two groups. Effective and efficient decision making processes are the most critical aspect of any application domain but particularly in healthcare, as the costs of erroneous decisions are huge in terms of human quality of life, and the resulting damage in most cases is irreversible. In this chapter, we outlined different aspects and challenges in providing effective clinical decision support systems, mostly from a technical view point. We discussed different models and approaches, information and knowledge representations, using data mining techniques to provide patterns and trends as additional type of clinical knowledge, challenges in future systems through customization of expert agents, and evaluation and acceptance of CDSS by users. However, as clinical decision systems are heavily user-oriented systems and clinicians are in general less technically oriented and overwhelmed by different types of information and workplace interruptions, user acceptance and adoption is very important to the success of such systems. We have included a section to overview the challenges in system evaluation, user adoption, and other related topics that consider the impact of human factors in the success of clinical decision support systems.

\section{References}

Abiteboul, S., Greenshpan, O. \& Milo, T. (2008). Modeling the mashup space, Information and Data Management, pp. 87-94.

ACM SIGCHI Curricula for Human-Computer Interaction (2011). http:/ /sigchi.org/cdg/.

Aleksovska-Stojkovska, L. \& Loskovska, S. (2010). Clinical decision support systems: Medical knowledge acquisition and representation methods, IEEE International Conference on Electro/Information Technology (EIT), pp. 1-6.

Anselma, L., Bottrighi, A., Molino, G. Montani, S., Terenziani, P. \& Torchio, M. (2011). Supporting knowledge-based decision making in the medical context: the GLARE approach, International Journal of Knowledge-Based Organizations. 1: 42-60.

Aspden, P., Mayhew, L. \& Rusnak, M. (1981). Dram: a model of health care resource allocation in czechoslovakia, omega - the international journal of management science 9(5): 509-518. 
Au, G. \& Paul, R. J. (1996). Visual interactive modelling: A pictorial simulation specification system, European Journal of Operational Research 91(1): 14-26.

Balas, E. A., Austin, S. M., Mitchell, J. A., Ewigman, B. G., Bopp, K. D. \& Brown, G. D. (1996). The clinical value of computerized information services: A review of 98 randomized clinical trials, Arch Fam Med 5(5): 271-278.

Balas, E. A. \& Boren, S. A. (2007). Clinical trials of information interventions, in E. S. Berner (ed.), Clinical Decision Support Systems, Health Informatics, Springer New York, pp. 140-155.

Berner, E. S. (2007). Clinical Decision Support Systems - Theory and Practice, Springer.

Berner, E. S. \& Lande, T. J. (2007). Overview of clinical decision support systems, in E. S. Berner (ed.), Clinical Decision Support Systems, Health Informatics, Springer New York, pp. 3-22.

Bodenreider, O. (2004). The Unified Medical Language System (UMLS): integrating biomedical terminology, Nucleic Acids Research 32(1): 267-270.

Bos, L., Marsh, A., Carroll, D., Gupta, S. \& Rees, M. (2008). Patient 2.0 empowerment, International Conference on Semantic Web and Web Services, pp. 164-167.

Collaboratory, I. (2004). Guideline interchange format (GLIF) 3.5 - technical specification.

Critchfield, G. \& Willard, K. (1986). Probabilistic analysis of decision trees using monte carlo simulation, Medical Decision Making 6: 85-92.

DMG (2010). Predictive model markup language (PMML) version 4.x specification. http:/ /www.dmg.org/pmml-v4-0-1.html.

Eben-Chaime, M. \& Pliskin, J. (1992). Incorporating patient travel times in decisions about size and location of dialysis facilities, Medical Decision Making 12: 44-51.

Eom, S. \& Kim, E. (2006). A survey of decision support system applications, Journal of the Operational Research Society 57: 1264-1278(15).

Formal concept analysis toolkit version 1.3 (2009). http:/ / sourceforge.net/projects/conexp.

Garg, A. X., Adhikari, N. K. J., McDonald, H., Rosas-Arellano, M. P., Devereaux, P. J., Beyene, J., Sam, J. \& Haynes, R. B. (2005). Effects of Computerized Clinical Decision Support Systems on Practitioner Performance and Patient Outcomes, JAMA: The Journal of the American Medical Association 293(10): 1223-1238.

Hall, R. E. (2010). Forward-Looking Decision Making: Dynamic Programming Models Applied to Health, Risk, Employment, and Financial Stability, Princeton University Press.

Hazen, G. B., Pellissier, J. M. \& Sounderpandian, J. (1998). Stochastic-tree models in medical decision making, Medical Decision Making 28: 64-80.

Health Level Seven Ballot (2011). http:/ / www.hl7.org/v3ballot/html/welcome/environment/ index.htm.

Health Level Seven official website (2011). http:/ / www.hl7.org.

Hershey, J. (1991). Sensitivity analysis in medical decision making: a direct line to linear programming, Medical Decision Making 11: 57-60.

HL7 (1999). Message development framework. version 3.3.

HL7 (2005). The clinical document architecture (CDA) standard specification. version 2.0.

IBM Mash up Center (2011). http:/ /www-01.ibm.com/software/info/mashup-center/.

Jayaratna, P. \& Sartipi, K. (2009). Tool-assisted Healthcare Knowledge to HL7 Message Translation, International Conference on Complex Medical Engineering, pp. 1-7.

Johnston, M. E., Langton, K. B., Haynes, R. B. \& Mathieu, A. (1994). Effects of Computer-based Clinical Decision Support Systems on Clinician Performance and Patient Outcome: A Critical Appraisal of Research, Annals of Internal Medicine 120(2): 135-142. 
Kaplan, B. (2001). Evaluating informatics applications - some alternative approaches: Theory, social interactionism, and call for methodological pluralism, International Journal of Medical Informatics 64: 39-56.

Kazemi, A., Fors, U. G., Tofighi, S., Tessma, M. \& Ellenius, J. (2010). Physician order entry or nurse order entry? comparison of two implementation strategies for a computerized order entry system aimed at reducing dosing medication errors, Journal of Medical Internet Research 12(1): e5.

Keen, P. \& Morton, M. S. (1978). Decision Support Systems: An Organizational Perspective, Addison-Wesley.

Kong, G., Xu, D.-L. \& Yang, J.-B. (2008). Clinical decision support systems: A review on knowledge representation and inference under uncertainties, International Journal of Computational Intelligence Systems 1: 159-167.

Kushniruk, A. W. \& Patel, V. L. (2004). Cognitive and usability engineering methods for the evaluation of clinical information systems, Journal of Biomedical Informatics 37: 56-76.

Leslie, S. J., Hartswood, M., Meurig, C., McKee, S. P., Slack, R., Procter, R. \& Denvir, M. A. (2005). Clinical decision support software for management of chronic heart failure: Development and evaluation, Computers in Biology and Medicine 36: 495-506.

Mandell, G., Bennett, J. \& Dolin, R. (2004). Principles and Practice of Infectious Diseases: 2-Volume, Churchill Livingstone.

Mangalampalli, A., Mangalampalli, S. M., Chakravarthy, R. \& Jain, A. K. (2006). A neural network based clinical decision-support system for efficient diagnosis and fuzzy-based prescription of gynecological diseases using homoeopathic medicinal system, Expert Systems with Applications 30: 109-116.

Najafi, M., Aghtar, S., Sartipi, K. \& Archer, N. (2011). Virtual remote nursing system, IEEE International Workshop on Consumer eHealth Platforms, Services and Applications, pp. 15.

Najafi, M. \& Sartipi, K. (2010). Client-side service composition using generic service representative, Proceedings of the 2010 Conference of the Center for Advanced Studies on Collaborative Research, pp. 238-252.

Oh, Y. H. \& Hwang, H. (2005). Deterministic inventory models for recycling system, Intelligent Manufacturing 17(4): 423-428.

Open Clinical: PROforma website (2011). http:/ / www.openclinical.org/gmm-proforma.html.

Oreilly Web 2.0 Books (2011). http:/ / oreilly.com/web2/.

Parsons, S., Gymtrasiewicz, P. \& Wooldridge, M. (eds) (2002). Game Theory and Decision Theory in Agent-Based Systems, Springer. Collection of papers.

Patrick, J. \& Puterman, M. L. (2006). Improving resource utilization for diagnostic services through flexible inpatient scheduling: A method for improving resource utilization, Journal of Operational Research Society 58(2): 235-245.

Perreault, L. \& Metzger, J. (1999). A pragmatic framework for understanding clinical decision support, Journal of Healthcare Information Management 13: 5-21.

ProgrammableWeb Web site (2011). http:/ / www.programmableweb.com/.

Protege website (2011). http:/ / protege.stanford.edu/.

Sandell, P. (2007). Framework for securing personal health data in clinical decision support systems, Journal of Healthcare Information Management 21(3): 34-40.

Santibanez, P., Chow, V., French, J., Puterman, M. \& Tyldesley, S. (2009). Reducing patient wait times and improving resource utilization at british columbia cancer agency's 
ambulatory care unit through simulation, Journal of Health Care Management Science 12(4): 392-407.

Schmidt, A. (2002). Ubiquitous computing-computing in context. PhD Thesis. Lancaster University, U.K.

Sherafat, R. \& K. Sartipi, P. J. (2010). A framework for data and mined-knowledge interoperability in clinical decision support systems, International Journal of Healthcare Information Systems and Informatics 5(1): 37 - 60.

Shim, J. P., Warkentin, M., Courtney, J. F., Power, D. J., Sharda, R. \& Carlsson, C. (2002). Past, present, and future of decision support technology, Decision Support Systems 33(2): $111-126$.

Sonnenberg, F. \& Beck, J. (1993). Markov models in medical decision making: A practical guide, Medical Decision Making 13: 322-38.

Systematized Nomenclature of Medicine Clinical Terminology (SNOMED CT) (2011). http://www. snomed.org/snomedct/index.html.

Testi, A. \& Tànfani, E. (2009). Tactical and operational decisions for operating room planning: Efficiency and welfare implications, Health Care Management Science 12: 363-373.

Trafton, J., Martins, S., Michel, M., Lewis, E., Wang, D., Combs, A., Scates, N., Tu, S. \& Goldstein, M. K. (2010). Evaluation of the acceptability and usability of a decision support system to encourage safe and effective use of opioid therapy for chronic, noncancer pain by primary care providers, Pain Medicine 11: 575-585.

Tsumoto, S., Hirano, S., Abe, H., Nakakuni, H. \& Hanada, E. (2005). Clinical decision support based on mobile telecommunication systems, Web Intelligence, IEEE / WIC / ACM International Conference on 0: 700-703.

Verlaene, K., Joosen, W. \& Verbaeten, P. (2007). Arriclides: An architecture integrating clinical decision support models, Proceedings of the 40th Annual Hawaii International Conference on System Sciences, pp. 1-10.

Yousefi, A., Mastouri, N. \& Sartipi, K. (2009). Scenario-oriented information extraction from electronic health records, IEEE International Symposium on Computer-Based Medical Systems, pp. $1-5$.

Zellner, B., Rand, S., Prost, R., Krouwer, H. \& Chetty, V. (2004). A cost-minimizing diagnostic methodology for discrimination between neoplastic and non-neoplastic brain lesions: utilizing a genetic algorithm, Academic Radiology 11(2): 169-177. 


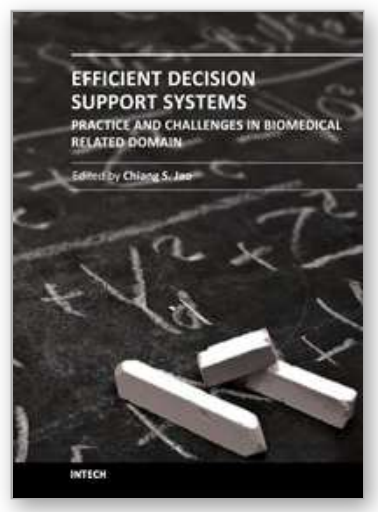

\author{
Efficient Decision Support Systems - Practice and Challenges in \\ Biomedical Related Domain \\ Edited by Prof. Chiang Jao
}

ISBN 978-953-307-258-6

Hard cover, 328 pages

Publisher InTeh

Published online 06, September, 2011

Published in print edition September, 2011

This series is directed to diverse managerial professionals who are leading the transformation of individual domains by using expert information and domain knowledge to drive decision support systems (DSSs). The series offers a broad range of subjects addressed in specific areas such as health care, business management, banking, agriculture, environmental improvement, natural resource and spatial management, aviation administration, and hybrid applications of information technology aimed to interdisciplinary issues. This book series is composed of three volumes: Volume 1 consists of general concepts and methodology of DSSs; Volume 2 consists of applications of DSSs in the biomedical domain; Volume 3 consists of hybrid applications of DSSs in multidisciplinary domains. The book is shaped decision support strategies in the new infrastructure that assists the readers in full use of the creative technology to manipulate input data and to transform information into useful decisions for decision makers.

\title{
How to reference
}

In order to correctly reference this scholarly work, feel free to copy and paste the following:

Kamran Sartipi, Norman P. Archer and Mohammad H. Yarmand (2011). Challenges in Developing Effective Clinical Decision Support Systems, Efficient Decision Support Systems - Practice and Challenges in Biomedical Related Domain, Prof. Chiang Jao (Ed.), ISBN: 978-953-307-258-6, InTech, Available from:

http://www.intechopen.com/books/efficient-decision-support-systems-practice-and-challenges-in-biomedicalrelated-domain/challenges-in-developing-effective-clinical-decision-support-systems 1

\section{INTECH}

open science | open minds

\section{InTech Europe}

University Campus STeP Ri

Slavka Krautzeka 83/A

51000 Rijeka, Croatia

Phone: +385 (51) 770447

Fax: +385 (51) 686166

www.intechopen.com

\section{InTech China}

Unit 405, Office Block, Hotel Equatorial Shanghai

No.65, Yan An Road (West), Shanghai, 200040, China

中国上海市延安西路65号上海国际贵都大饭店办公楼 405 单元

Phone: +86-21-62489820

Fax: $+86-21-62489821$ 
(C) 2011 The Author(s). Licensee IntechOpen. This chapter is distributed under the terms of the Creative Commons Attribution-NonCommercialShareAlike-3.0 License, which permits use, distribution and reproduction for non-commercial purposes, provided the original is properly cited and derivative works building on this content are distributed under the same license. 